TAO, Vol. 15, No. 4, 727-741, November 2004

\title{
NOTES AND CORRESPONDENCE
}

\section{Source Parameters of Regional Earthquakes in Taiwan: January-December, 2002}

\author{
Wen-Tzong Liang ${ }^{1, *}$, Yu-Hua Liu ${ }^{1}$, and Honn Kao ${ }^{1,2}$ \\ (Manuscript received 16 March 2004, in final form 23 July 2004)
}

\begin{abstract}
We report on the source parameters of 194 earthquakes that occurred between January and December 2002, in the Taiwan region. The improved CMT inversion algorithm by Kao et al. (1998) is used to overcome the generally higher background noise and the heterogeneous velocity structure resulting from the complex tectonic interactions near Taiwan. To make the results more accessible and useful to the academic community, both the table that summarizes the reported source parameters and the complete set of inversion results are available electronically from the BATS worldwide web site at http://bats.earth.sinica.edu.tw/CMT_Solutions/cmtF2002. html.
\end{abstract}

(Key words: Broadband Array in Taiwan for Seismology, Earthquake source parameters, Waveform inversion, Taiwan)

Successful development of the centroid-moment-tensor (CMT) inversion technique in the early 1980's has enabled the systematic determination of source parameters for global large and moderately-sized earthquakes (e.g., Dziewonski et al. 1981; Kawakatsu et al. 1995; Sipkin 1982). The CMT inversion technique was later successfully applied to regional earthquakes because of the increasing knowledge of detailed velocity structures on a regional scale and the establishment of regional broadband networks (e.g., Dreger and Helmberger 1993; Fan and Wallace 1995; Lay et al. 1994; Thio and Kanamori 1995; Zhao and Helmberger 1994). Consequently, the routine CMT solution reports for smaller, regional earthquakes has become

\footnotetext{
1 Institute of Earth Sciences, Academia Sinica, Taipei, Taiwan, ROC

2 Pacific Geoscience Centre, Geological Survey of Canada, Canada

* Corresponding author address: Dr. Wen-Tzong Liang, Institute of Earth Sciences, Academia Sinica, Taipei, Taiwan, ROC;E-mail: wtl@earth.sinica.edu.tw
} 
standard practice for many regional broadband seismographic networks (e.g., Zhu and Helmberger 1996; Pasysnos et al. 1996).

Establishment of the "Broadband Array in Taiwan for Seismology (BATS)" has enabled the systematic determination of reliable source parameters for regional earthquakes in Taiwan through the CMT technique (Kao et al. 1998). The network began its test operation in late 1994 (Fig. 1). A brief description of the BATS configuration and operation can be found in Kao et al. $(1998,2001)$ and Kao and Jian $(1999,2001)$. The Data Management Center of the

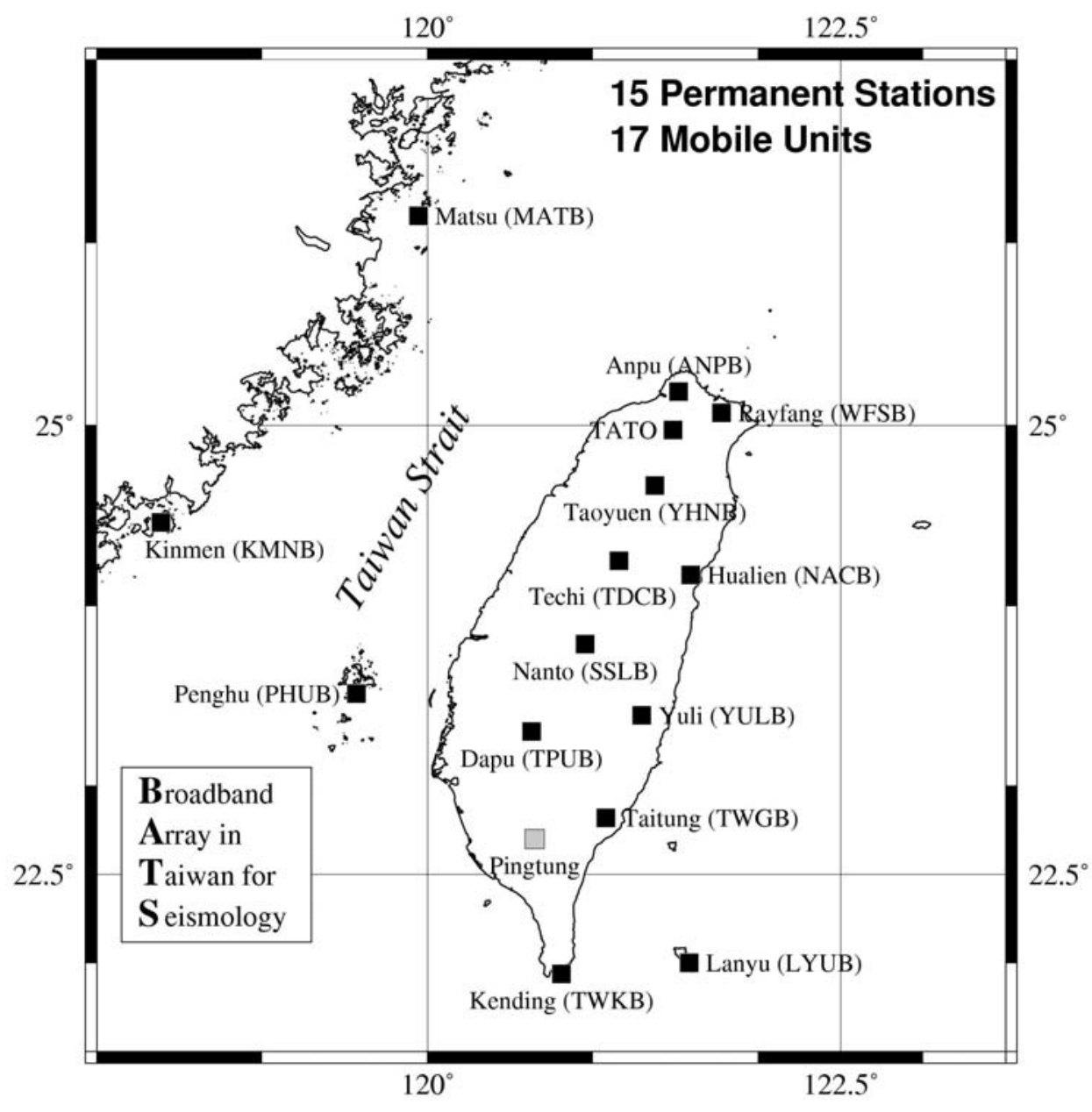

Fig. 1. Map of the "Broadband Array in Taiwan for Seismology (BATS)". Solid and gray squares show stations currently in operation and under construction, respectively. In addition to the permanent stations, BATS includes 17 portable stations that can be deployed for specific research tasks. 
Institute of Earth Sciences (DMC-IES), Academia Sinica is responsible for BATS data archiving and distribution. One copy of BATS data is contributed to the Data Management Center of the Incorporated Research Institutions for Seismology (DMC, IRIS) for the same purpose. Interested readers can obtain updated information at BATS worldwide web site (http://bats.earth. sinica.edu.tw).

Our CMT algorithm begins with a background-noise evaluation that determines the frequency band used in the inversion. The cut-off frequency of the lower corner is usually at $\sim 0.02 \mathrm{~Hz}$, which is determined by comparing the power spectra of waveform windows $300 \mathrm{~s}$ before and after the $\mathrm{P}$ first arrival (Kao et al. 1998). We also utilize a two-step procedure to allow different velocity models for different station-source pairs. This is to mimic the effect of heterogeneous velocity structures in the Taiwan region. For more technical details, readers are referred to our previous reports (Kao et al.1998; Kao and Jian 1999).

To characterize the inversion quality, each inversion result is classified using a letter (AF) and a digit (1-4) combination depending on two parameters: the waveform misfit (E) and the CLVD component amount $(\varepsilon)$, respectively. The derivations of $\mathrm{E}$ and $\varepsilon$ were presented in our previous reports (Kao et al. 1998; Kao and Jian 1999) and listed in Table 1.

Table 1. Quality classification of the inversion results.

\begin{tabular}{cc} 
Class & Criteria \\
\hline & Average Waveform Misfit (E) \\
A & $0 \leq \mathrm{E}<0.3$ \\
B & $0.3<\mathrm{E} \leq 0.5$ \\
C & $0.5<\mathrm{E} \leq 0.7$ \\
D & $0.7<\mathrm{E} \leq 0.9$ \\
E & $0.9<\mathrm{E} \leq 1.1$ \\
F & $\mathrm{E}>1.1$ \\
& CLVD component $(\varepsilon)$ \\
1 & $<\varepsilon \leq 0.1$ \\
2 & $0.1<\varepsilon \leq 0.25$ \\
3 & $0.25<\varepsilon \leq 0.4$ \\
4 & $\varepsilon>0.4$
\end{tabular}

Following the criteria defined in Kao and Jian (1999), we report on source parameters only if they meet the following criteria: (1) 3-component waveforms from at least three stations are used in the inversion, and (2) the inversion quality must be higher than $\mathrm{C} 4$. A total of 194 earthquakes that satisfy these conditions are listed in Table 2. The epicenters for all of these studied earthquakes are plotted in Figs. 2a, b and the corresponding moment-tensor solutions and focal depths are plotted in Fig. 2c. 


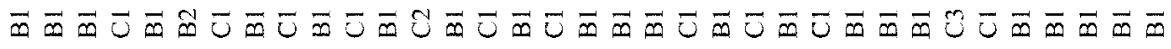

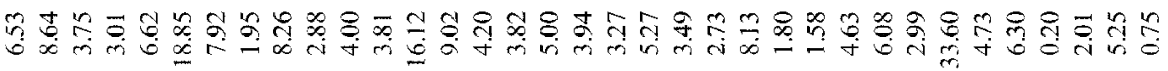

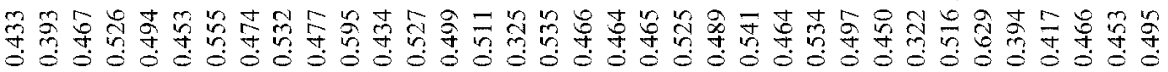

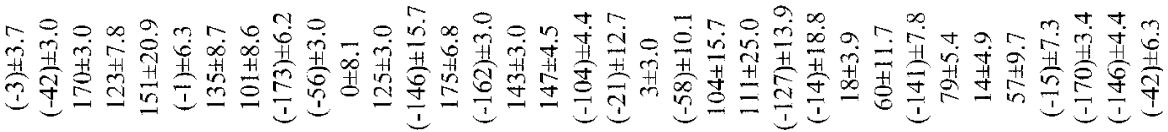

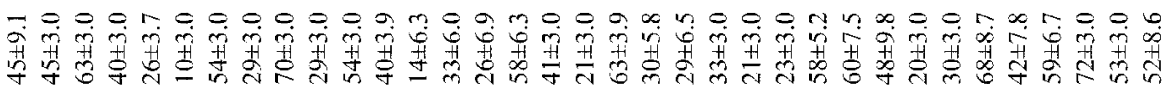

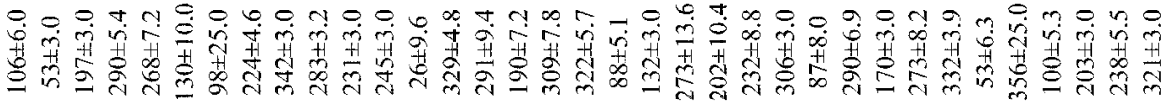

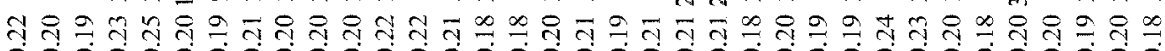

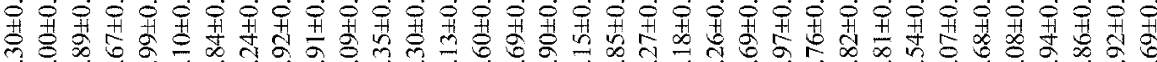

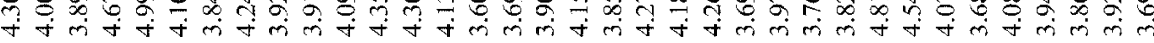

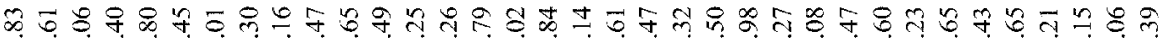

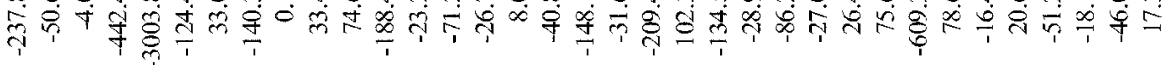

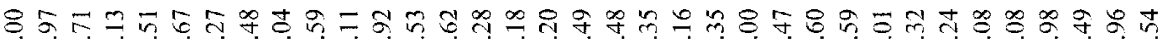

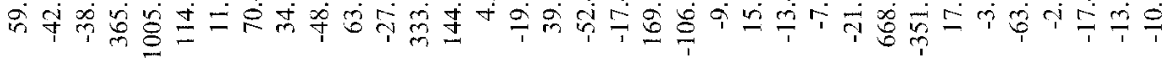

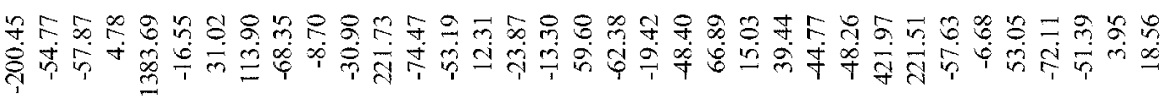

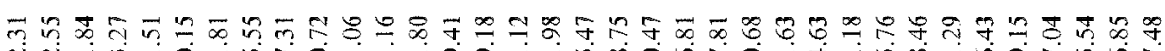

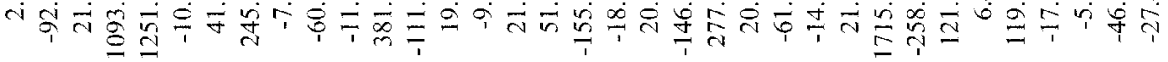

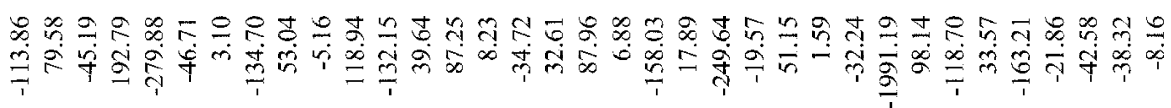

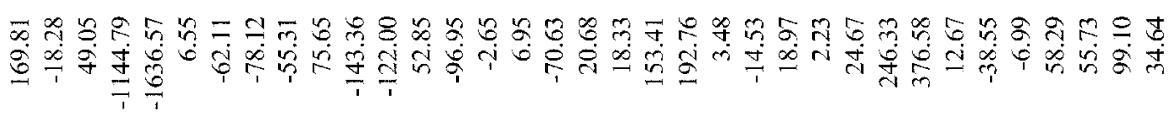

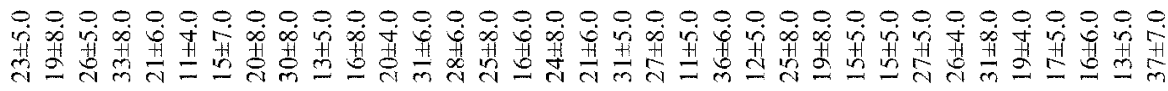

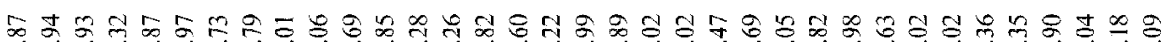

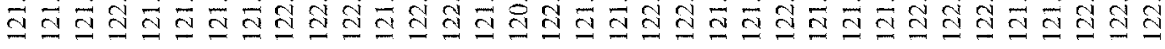

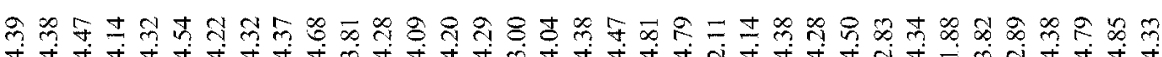

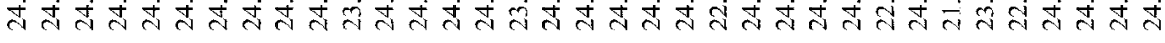

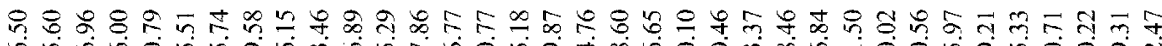

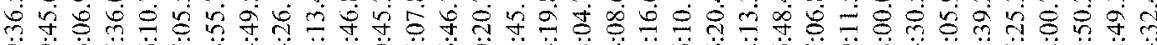

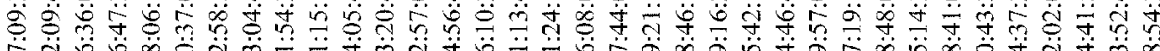

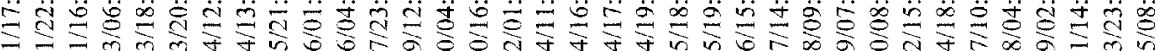

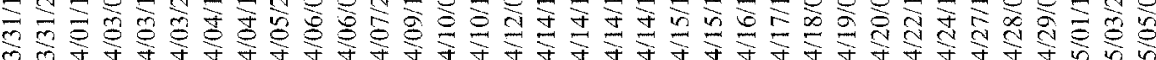

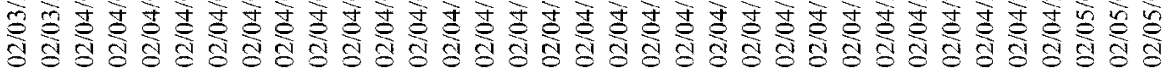

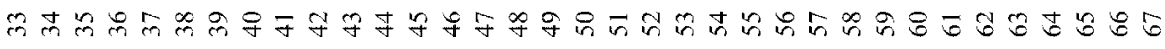




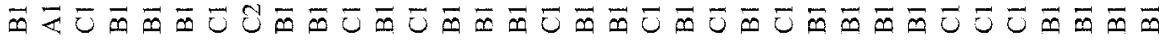

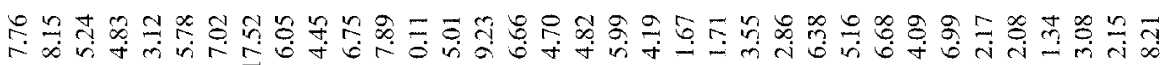

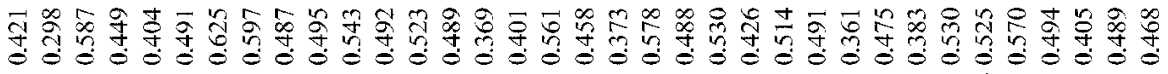

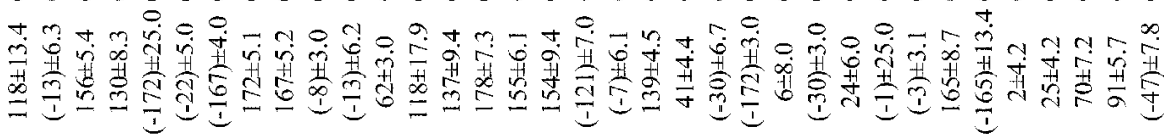

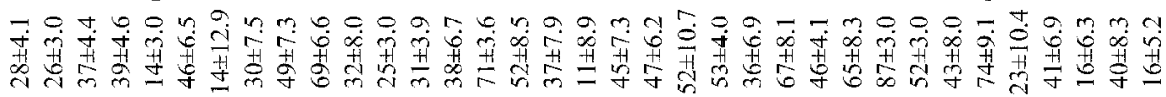

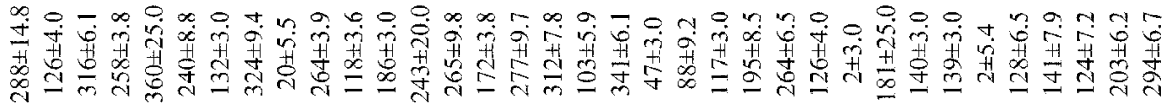

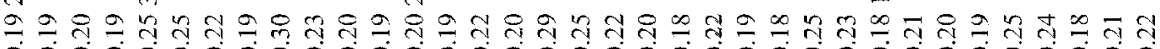

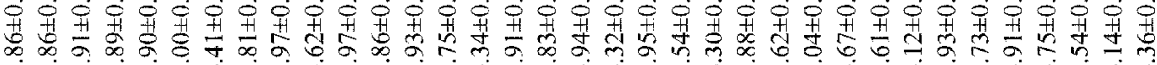

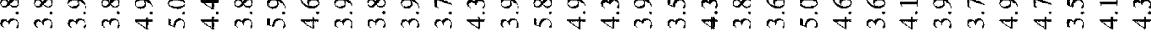

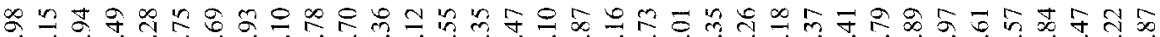

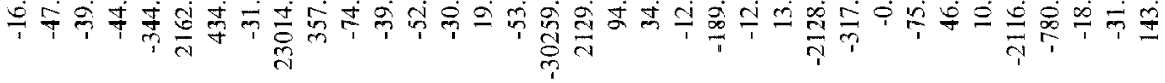

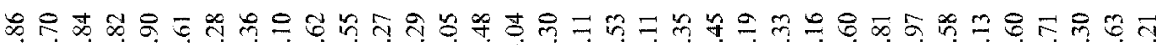

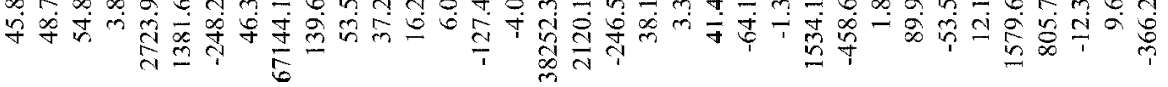

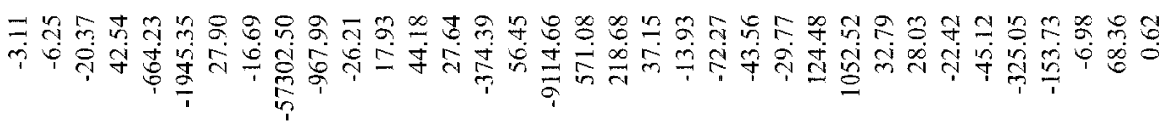

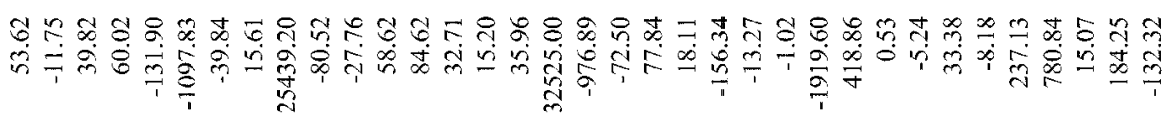

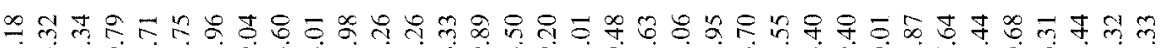

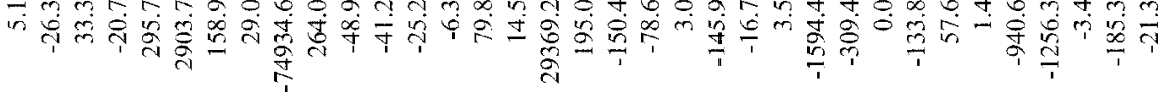

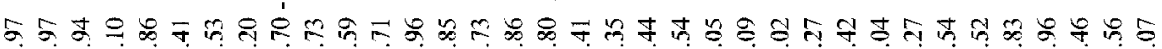

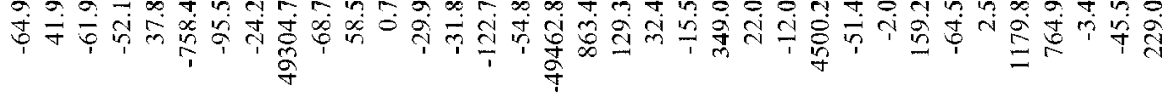

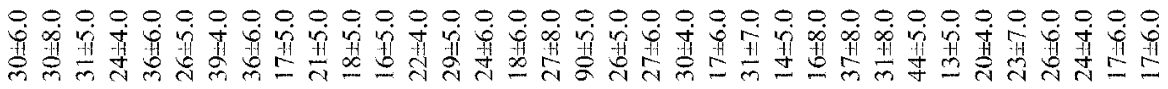

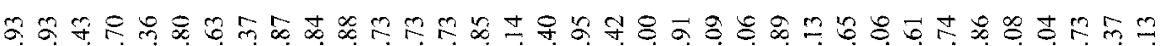

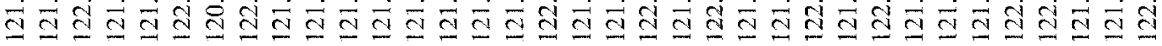

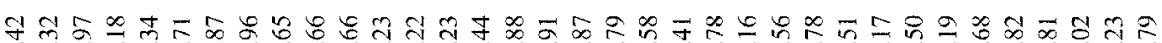

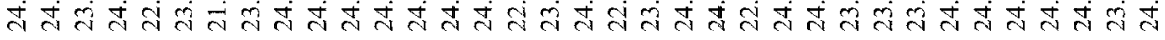
तु

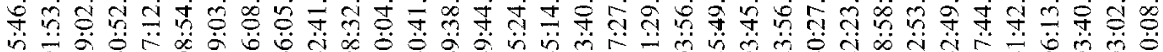

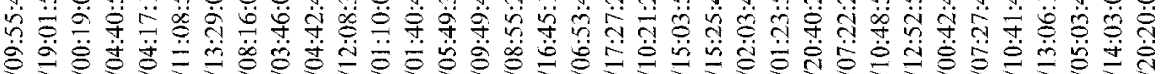

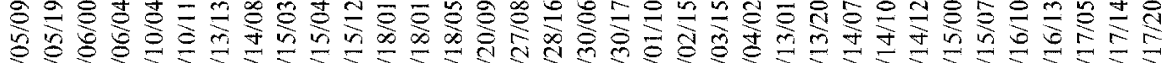

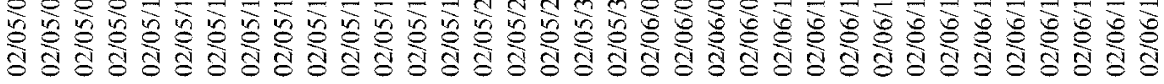

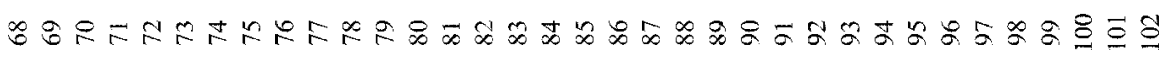




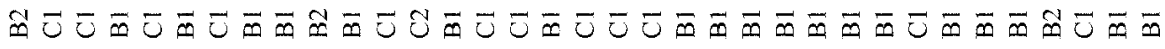

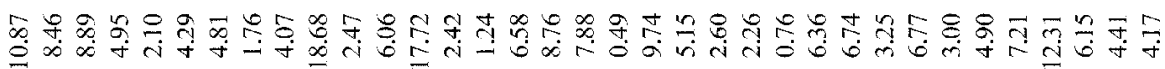

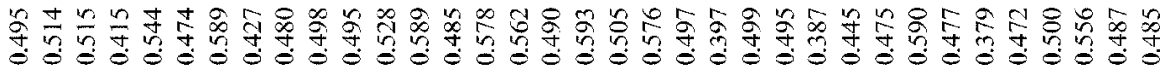

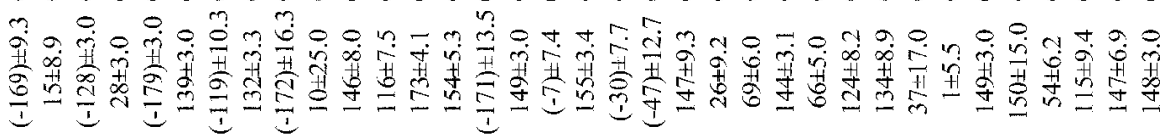

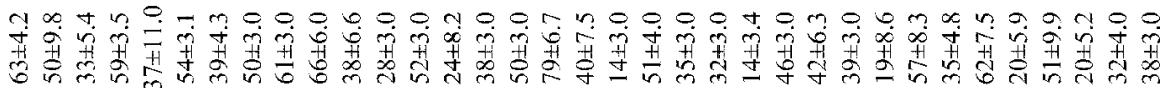

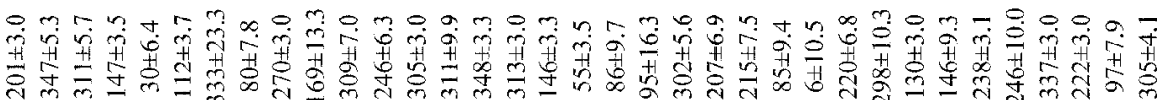
तथ 0 व

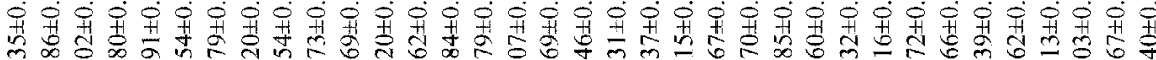

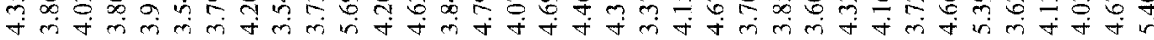

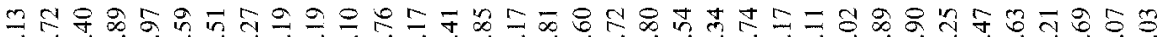

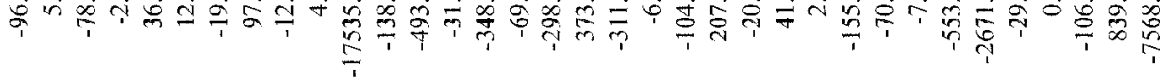

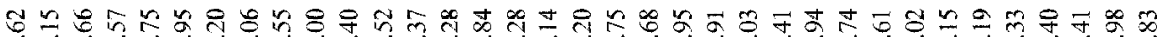

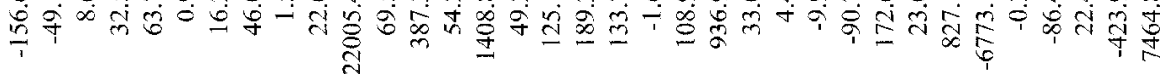

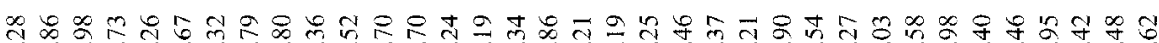

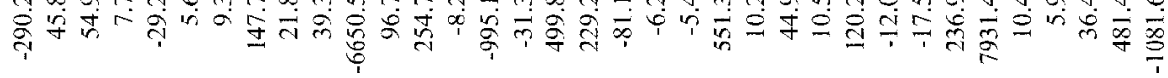

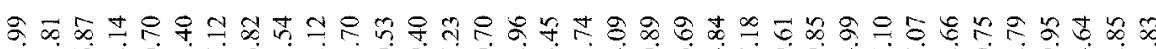

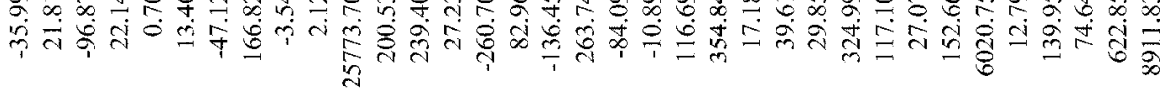

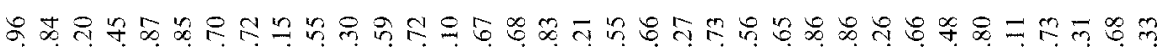

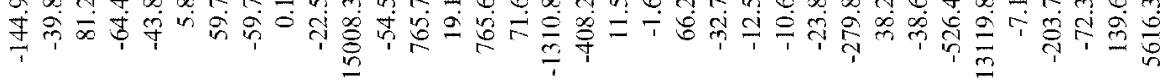

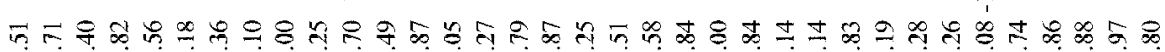

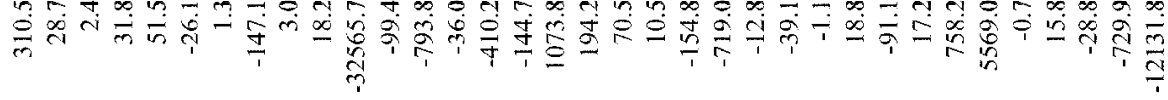

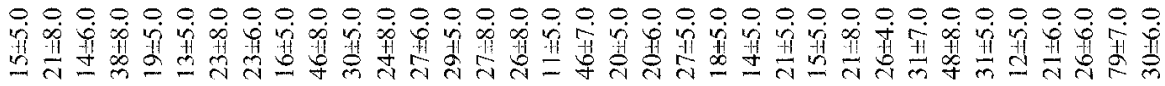

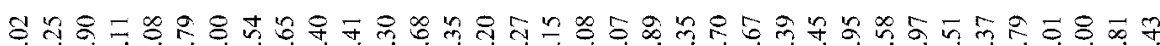

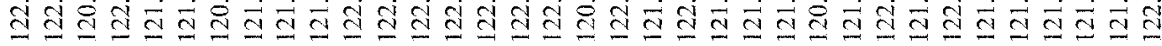

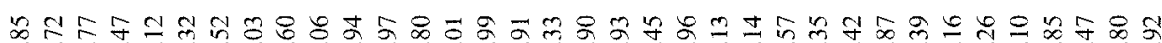

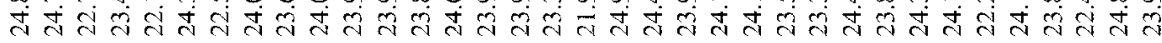
5

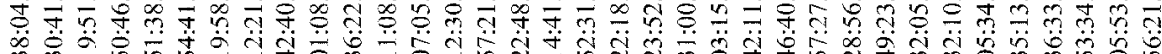

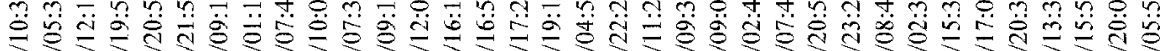

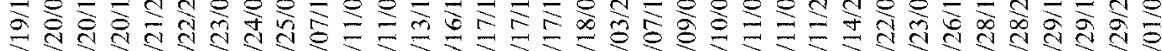

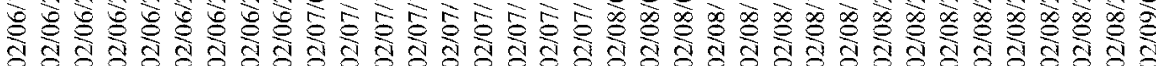

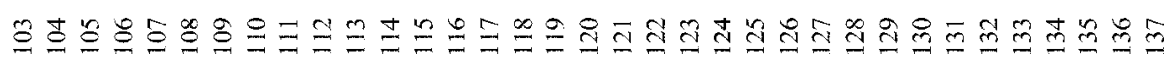




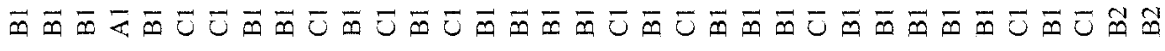

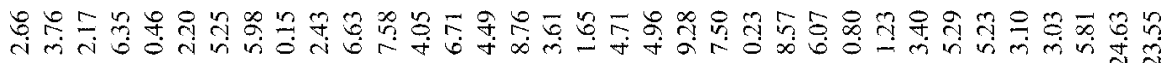

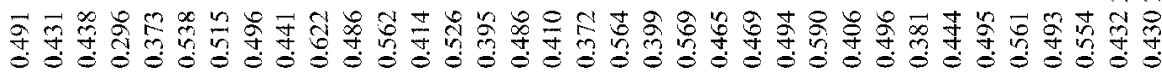
भ

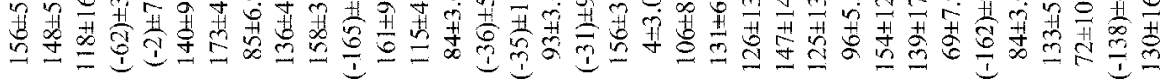

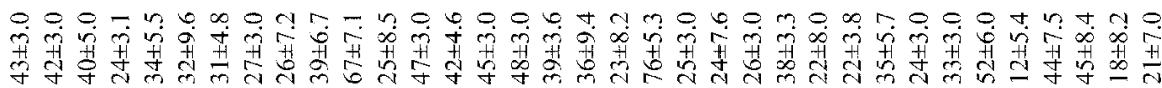

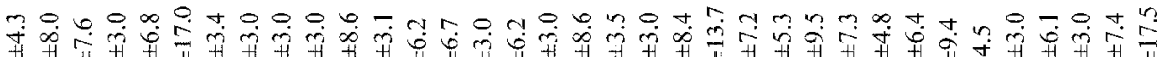

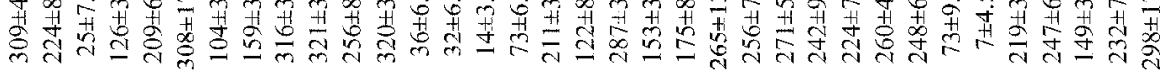

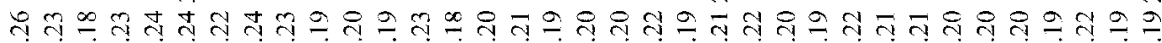

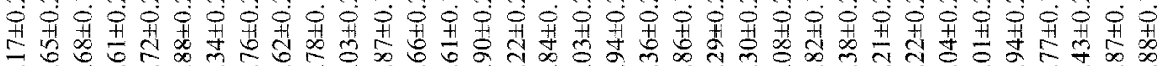

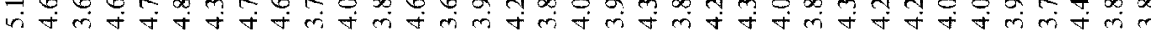

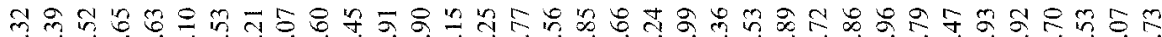

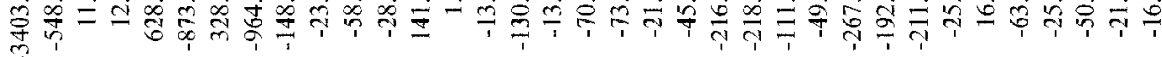

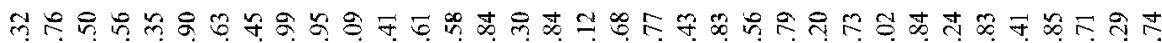

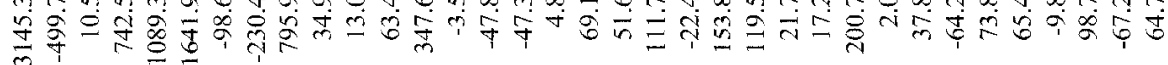

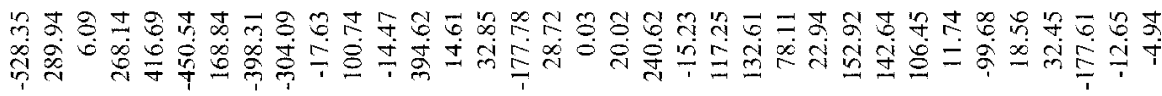

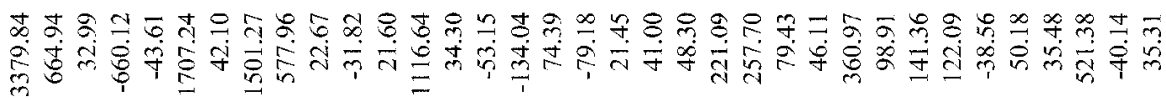

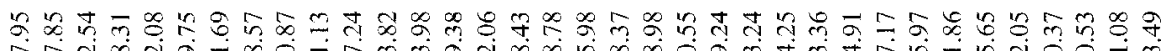

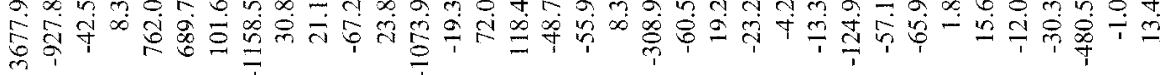

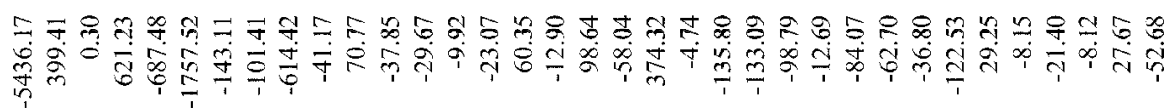

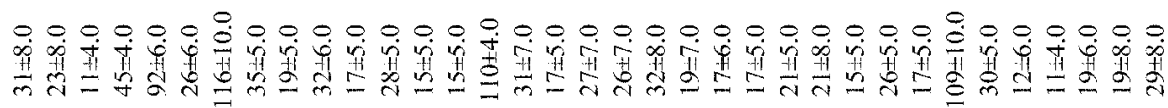

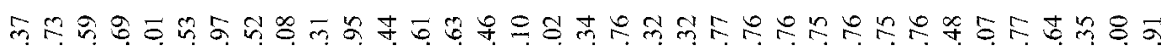

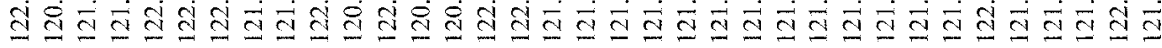
б.

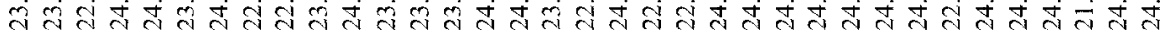

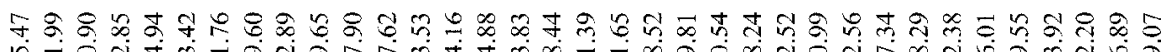

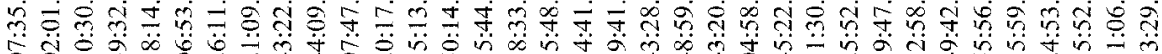

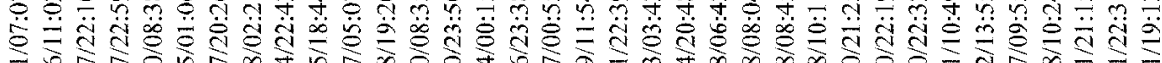

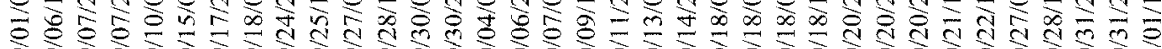

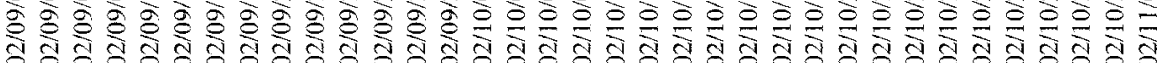

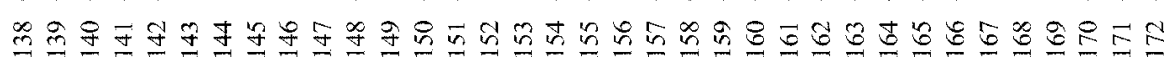




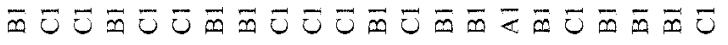

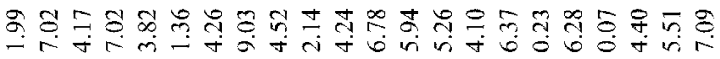

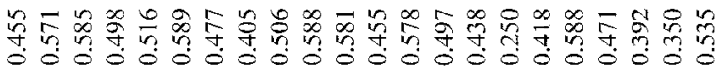

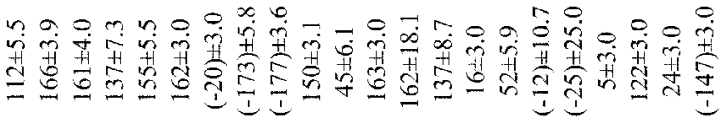

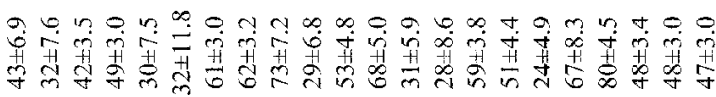

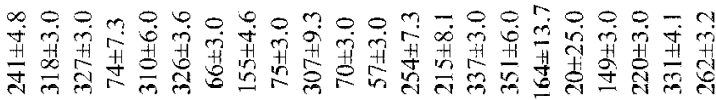

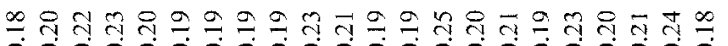

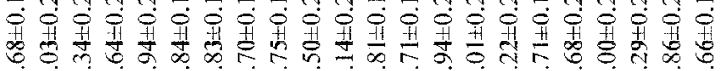

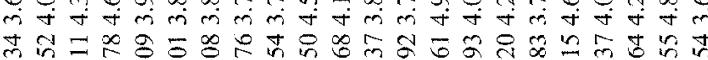

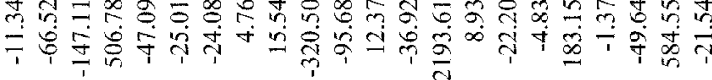

5푸유.

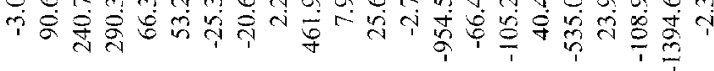

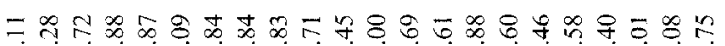

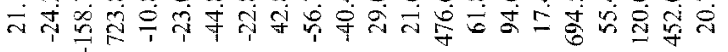

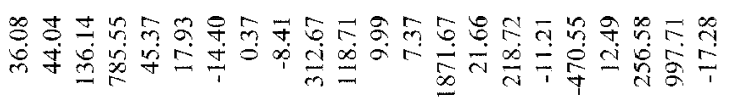

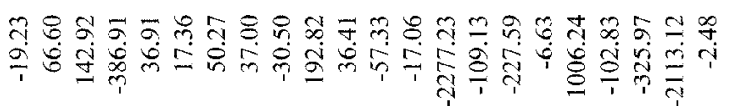

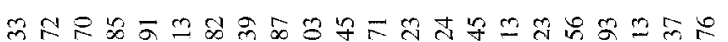
Tं

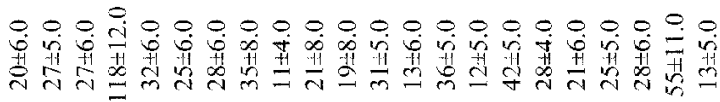
m 궁

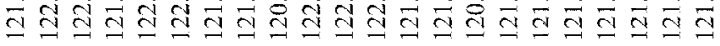

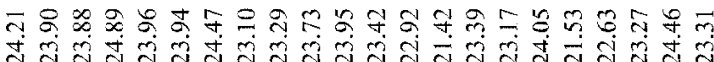

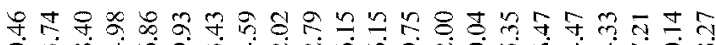

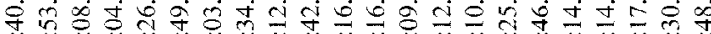

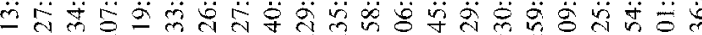

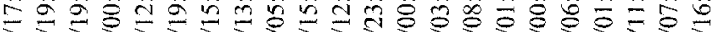

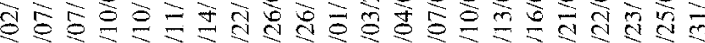

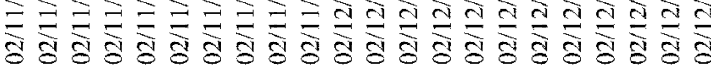




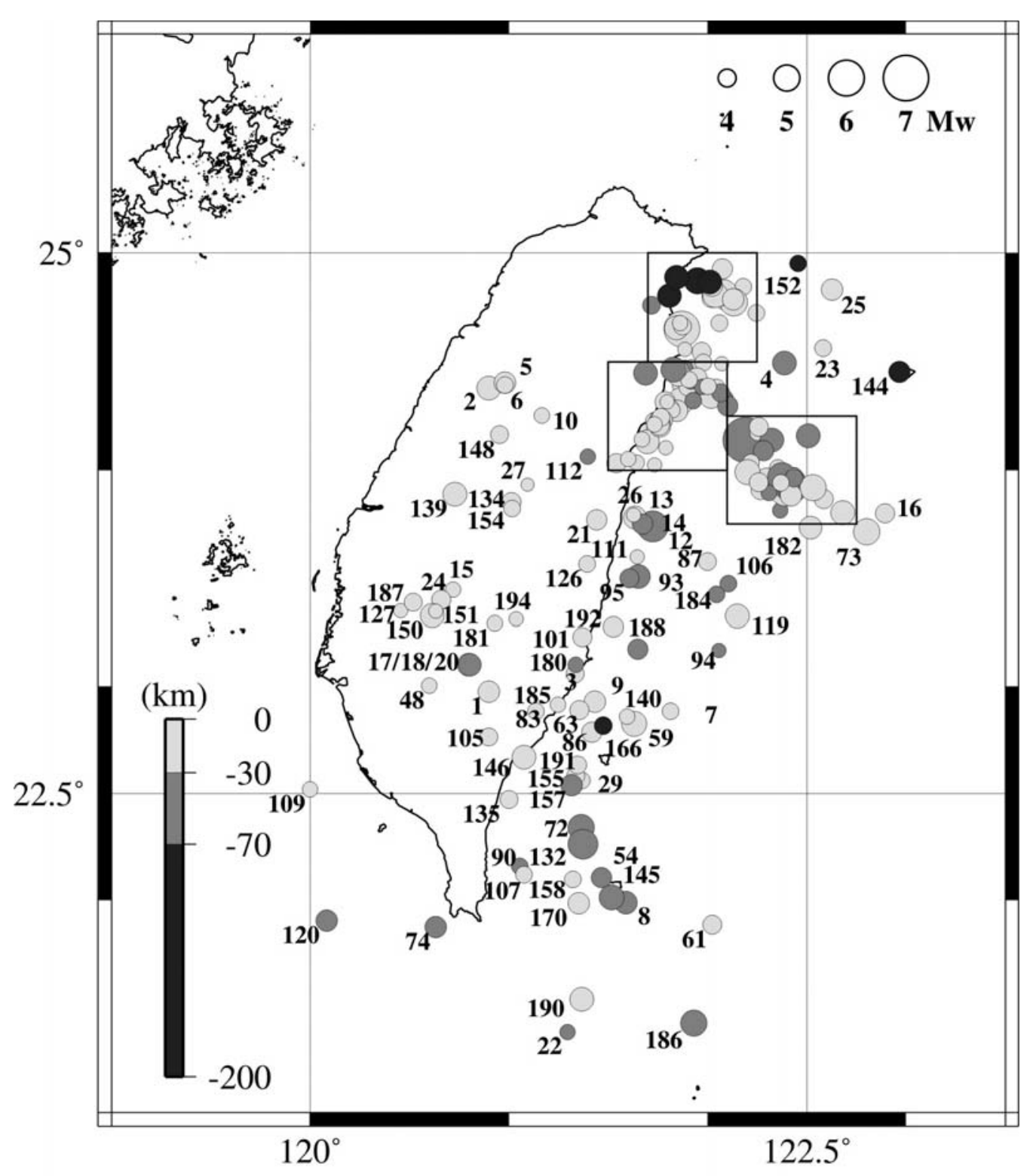

Fig. 2a. Moment-tensor inversion results. (a) Map showing the epicenters of 194 studied earthquakes. The size and shade of each symbol represent the corresponding moment magnitude and focal depth, respectively, as shown by the scales. Event numbers are according to Table 2 . 


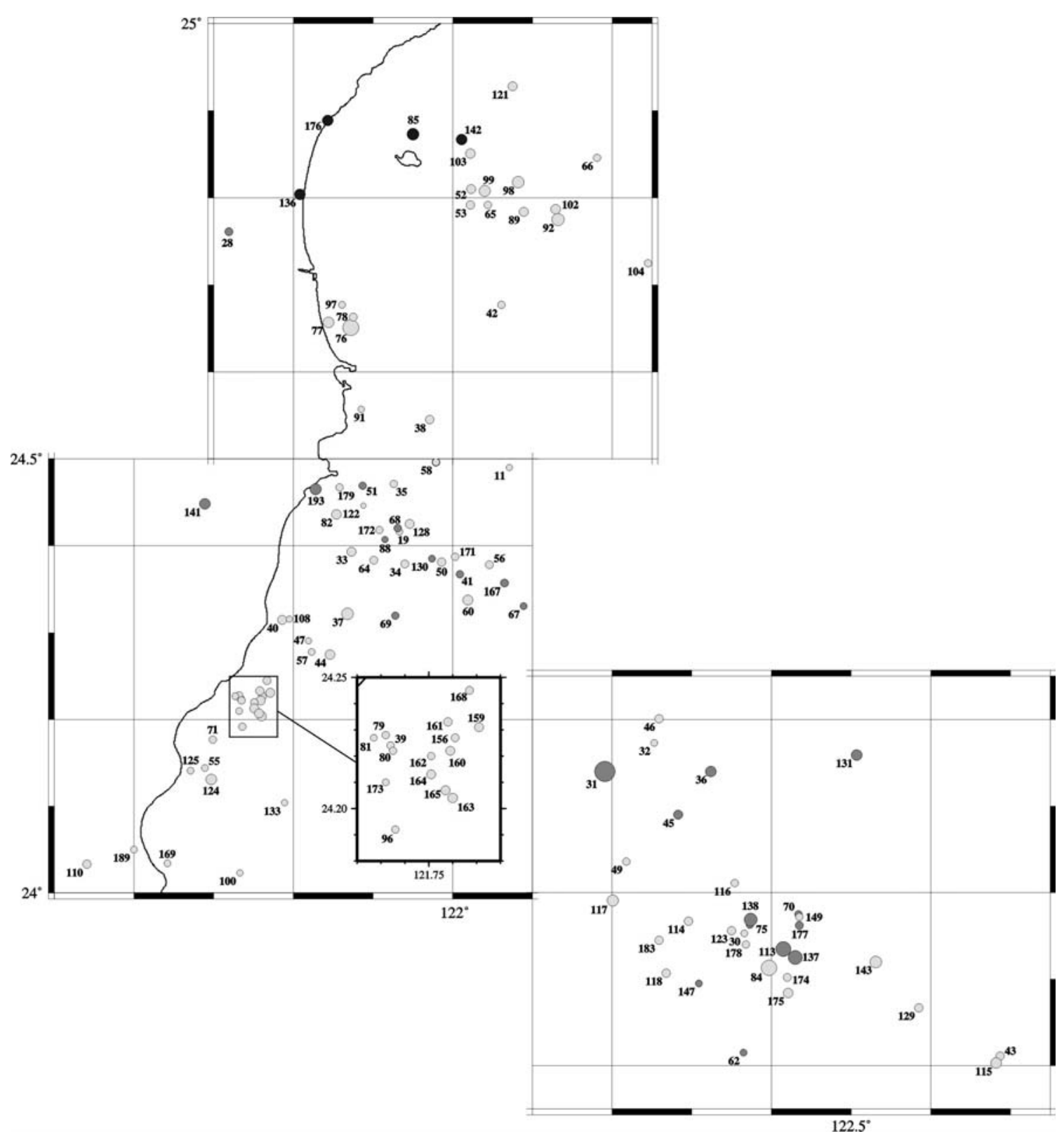

Fig. 2b. Moment-tensor inversion results. (b) A blow-up map to show the epicentral region of the March 31, 2002 sequence $\left(M_{w}=7.1\right)$. 
Fig. 2c. Moment-tensor inversion results. (c) Corresponding moment-tensor solutions. Darkened areas show quadrants with compressional P-wave first motions. $\mathrm{P}$ and $\mathrm{T}$ axes are marked by small solid and open circles, respectively. The corresponding best double couple solutions are shown by pairs of solid lines. The first number above each fault plane solution is the event number, whereas the number in parenthesis is the best focal depth. 


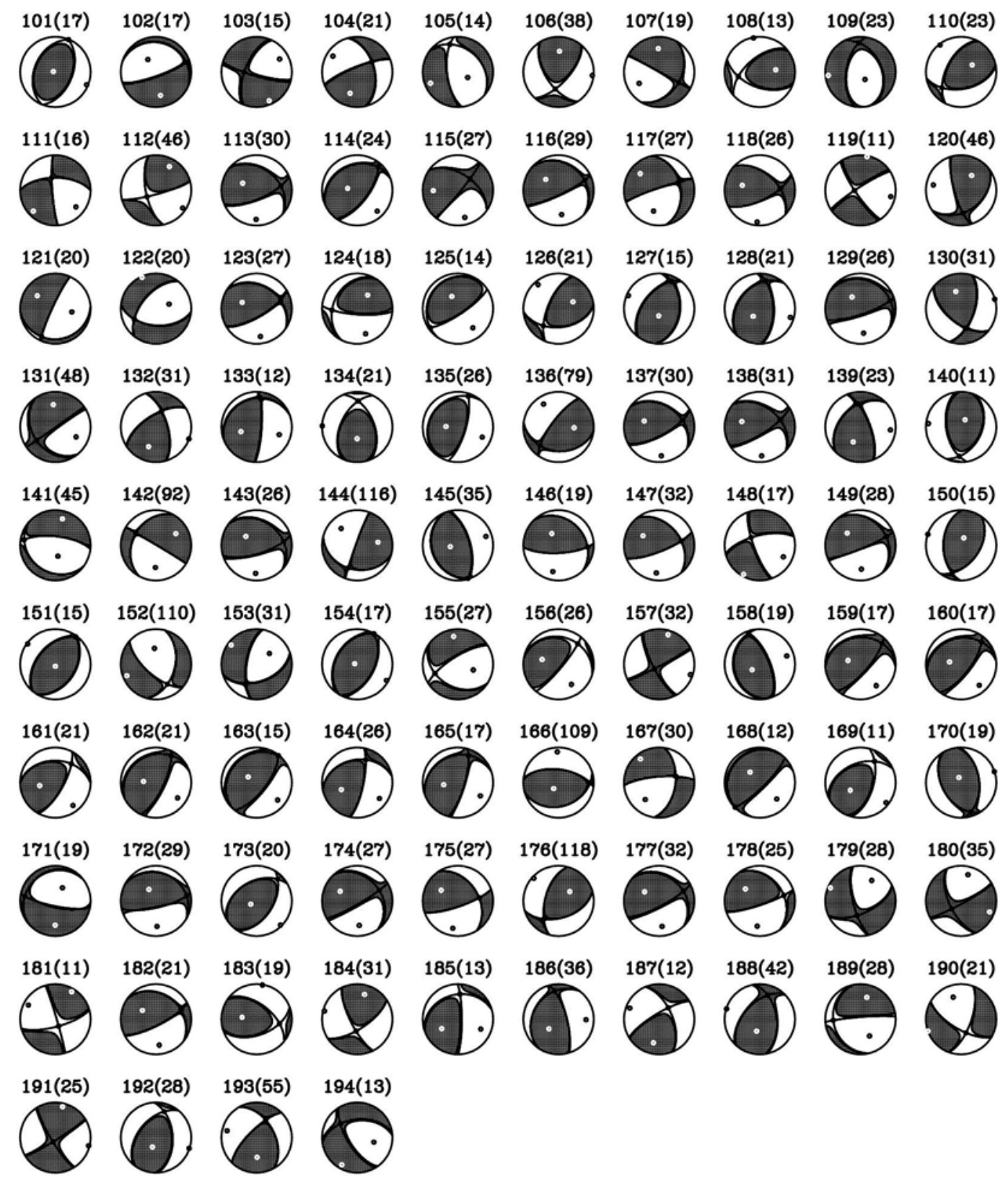

(Fig. 2c. continued) 
Due to practical concerns, the inversion results are presented as an electronic appendix to this report. Interested readers can download the complete set from the BATS worldwide web site at http://bats.earth.sinica.edu.tw/CMT_Solutions/cmtF2002.html. To make the results more accessible and useful to the academic community, Table 2 and the tables in our previous reports showing the source parameters of earthquakes between 1995 and 2001 (Kao and Jian 1999; Kao et al. 2001, 2002a, 2002b; Liang et al. 2003) are also available from our web site (http://bats.earth.sinica.edu.tw/CMT_Solutions/).

Acknowledgments Technical staff at IES led by Chun-Chi Liu assumes the primary responsibility for maintaining BATS.

\section{REFERENCES}

Dreger, D. S., and D. V. Helmberger, 1993: Determination of source parameters at regional distances with three-component sparse network data.J. Geophys. Res., 98, 8107-8125.

Dziewonski, A. M., T. A. Chou, and J. H. Woodhouse, 1981: Determination of earthquake source parameters from waveform data for studies of global and regional seismicity.J. Geophys. Res., 86, 2825-2852.

Fan, G., and T. Wallace, 1995: Focal mechanism of a recent event in South Africa: A study using a sparse very broadband network. Seismol. Res. Lett., 66, 13-18.

Kao, H., and P. R. Jian, 1999: Source parameters of regional earthquakes in Taiwan: July 1995-December 1996. TAO, 10, 585-604.

Kao, H., P. R. Jian, K. F. Ma, B. S. Huang, and C. C. Liu, 1998: Moment-tensor inversion for offshore earthquakes east of Taiwan and their implications to regional collision.Geophys. Res. Lett., 25, 3619-3622.

Kao, H., and P. R. Jian, 2001: Seismogenic patterns in the Taiwan region: Insights from source parameter inversion of BATS data. Tectonophys., 333, 179-198.

Kao, H., Y. H. Liu, and P. R. Jian, 2001: Source parameters of regional earthquakes in Taiwan: January-December 1997. TAO, 12, 431-439.

Kao, H., Y. H. Liu, S. C. Chen, and W. T. Liang, 2002: Source parameters of regional earthquakes in Taiwan: January-December, 1998. TAO, 13, 197-204.

Kao, H., Y. H. Liu, W. T. Liang and W. P. Chen, 2002: Source parameters of regional earthquakes in Taiwan: 1999-2000 Including the Chi-Chi earthquake sequence.TAO, 13, 279-298.

Kawakatsu, H., 1995: Automated near-realtime CMT inversion. Geophys. Res. Lett., 22, 25692572.

Lay, T., J. Ritsema, C. J. Ammon, and T. Wallace, 1994: Rapid source-mechanism analysis of the April 29, 1993 Cataract Creek $\left(\mathrm{M}_{\mathrm{w}}=5.3\right)$, northern Arizona earthquake. Bull. Seismol. Soc. Am., 84, 451-457. 
Liang, W. T, Y. H Liu, and H. Kao, 2003: Source parameters of regional earthquakes in Taiwan: January-December, 2001. TAO, 14, 249-260.

Pasyanos, M. E., D. S. Dreger, and B. Romanowicz, 1996: Toward real-time estimation of regional moment tensors. Bull. Seimol. Soc. Am., 86, 1255-1269.

Sipkin, S. A., 1982: Estimation of earthquake source parameters by the inversion of waveform data: synthetic waveforms. Phys. Earth Planet. Inter., 30, 242-259.

Thio, H. K., and H. Kanamori, 1995: Moment-tensor inversions for local earthquakes using surface waves recorded at TERRAscope. Bull. Seismol. Soc. Am., 85, 1021-1038.

Zhao, L. S., and D. V. Helmberger, 1994: Source estimation from broadband regional seismograms. Bull Seismol. Soc. Am., 84, 91-104.

Zhu, L., and D. V. Helmberger, 1996: Advancement in source estimation techniques using broadband regional seismograms. Bull. Seismol. Soc. Am., 86, 1634-1641. 